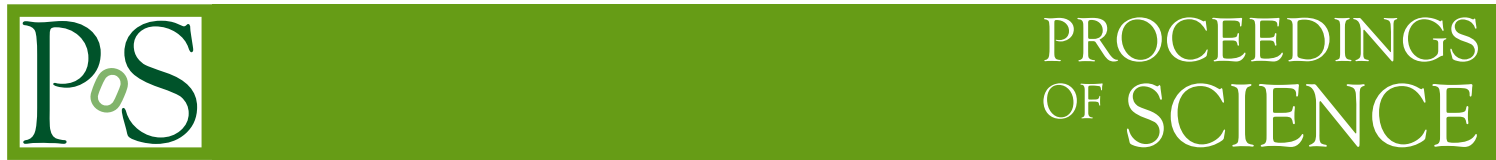

\title{
High Momentum Probes of Nuclear Matter
}

\section{Rainer Fries*}

Cyclotron Institute, Texas A\&M University, USA and RIKEN/BNL Research Center, USA

E-mail: rjfries@comp.tamu.edu

We discuss how the chemical composition of QCD jets is altered by final state interactions in surrounding nuclear matter. We describe this process through conversions of leading jet particles. We find that conversions lead to an enhancement of kaons at high transverse momentum in $\mathrm{Au}+\mathrm{Au}$ collisions at RHIC, while their azimuthal asymmetry $v_{2}$ is suppressed.

4th international workshop High-pT physics at LHC 09

February 4-7, 2009

Prague, Czech Republic

\footnotetext{
${ }^{*}$ Speaker.
} 
Jets of high energy particles can be used as probes to study dense and hot phases of nuclear matter [1, 2, 3, 4, 5, 6]. Experiments at the Relativistic Heavy Ion Collider (RHIC) have shown that quark gluon plasma just above the phase transition temperature is a very opaque substance. These measurements usually compare single particle spectra and two-particle correlations at intermediate and high transverse momenta $p_{T}$ in nuclear collisions to those in elementary $p+p$ collisions. The quantitative outcome of these studies are estimates for the transport coefficient $\hat{q}=\mu^{2} / \lambda$ in hot nuclear matter, the average momentum transfer squared per mean free path. Recently, it has been suggested that studying the yield of particles at high $p_{T}$ can yield much more information by adding the flavor of hadrons as an additional observable [7].

Obviously, fast quarks and gluons passing through nuclear matter can change identity through conversion processes. In other words, the "flavor" (loosely used as a notation for the identity of a particle) of a leading jet parton is not conserved. This has a variety of observable consequences. Let us first point out that the rate at which conversions between different flavors occur depends mainly on the total cross section of a conversion channel. It is therefore sensitive to the mean free path $\lambda$ of a particle, and the chemical composition of the medium, rather than to the transport coefficient $\hat{q}$.

It was first pointed out in 2002 that light quarks and gluons passing through quark gluon plasma can "convert" into real and virtual photons through Compton and annihilation processes with thermal partons, $q+\bar{q} \rightarrow \gamma+g$ and $g+q \rightarrow \gamma+q$, resp. [8]. Here, we use a notation where the momentum of the first particle mentioned on both sides of the reaction equation is much larger than the momentum of the second particle in the lab frame and it is therefore considered to be either the leading parton of a jet or another high- $p_{T}$ particle. This source of photons has been studied by several authors since $[9,10,11,12,13]$. It appears to make a non-negligible contribution to the total direct photon spectrum for transverse momenta of several $\mathrm{GeV} / c$.

Flavor changing channels and conversions for gluons into quarks and vice versa, $q+g \leftrightarrow g+q$ and $q+\bar{q} \leftrightarrow g+g$, have been discussed by several authors $[14,15,16]$ in the past. Recent interest in this process was fueled by the idea that partonic energy loss predicts a color factor $9 / 4$ for
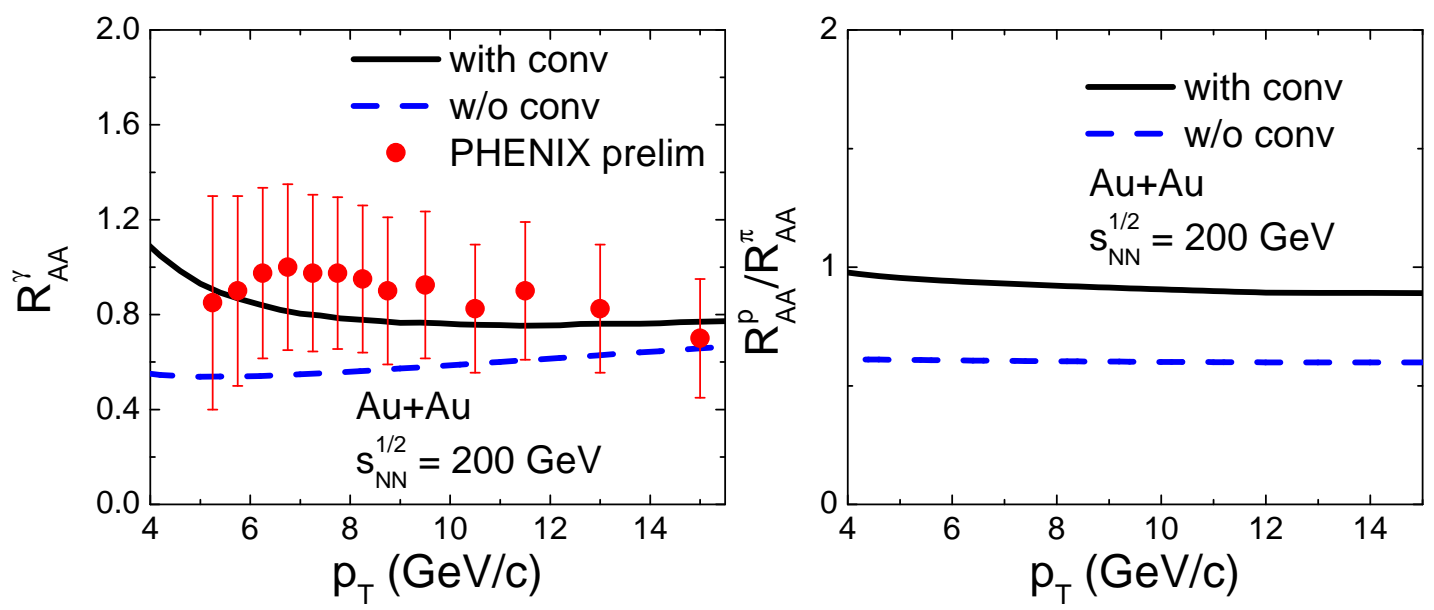

Figure 1: Left panel: The nuclear modification factor $R_{A A}$ for direct photons with and without conversions switched on, calculated in the model introduced in [7] (preliminary PHENIX data from [17]). Right panel: The ratio of nuclear modification factors for protons and pions is approaching one if conversions are allowed. 


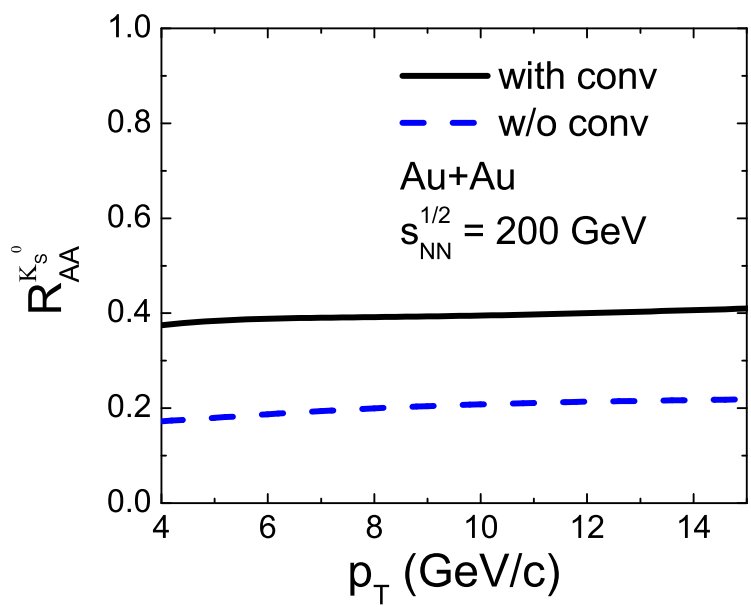

Figure 2: $R_{A A}$ for neutral kaons with and without conversion processes allowed. The strangeness in the jet sample is driven towards equilibrium by coupling it chemically to the quark gluon plasma.

the relative quenching strength of gluons and quarks. This could in principle translate into an observable difference in quenching between various hadron species, depending on the branching ratios of partons into hadrons via the fragmentation process. A primary candidate to look for this signature is the proton over pion ratio. In some state-of-the-art fragmentation functions like the AKK set [18] protons have a large contribution from gluon fragmentation, leading to larger suppression of protons in this picture due to stronger gluon quenching. No such suppression was found by the STAR experiment [19]. A lack of suppression would still be consistent with a partonic origin of energy loss if conversions are taken into account. A typical high- $p_{T}$ particle on its way out of the fireball could change identity several times between quark and gluon, leading to a rapid fading of the difference in color factors for any observable. As a word of caution, it is clear that the interpretation of this particular observable has a large uncertainty due to the input from fragmentation functions.

Examples for the nuclear modification factor of photon (left) and the ratio of proton and pion nuclear modification factors $R_{A A}$ (right) can be found in Fig. 1. Here, conversions were modeled by propagating leading jet partons through a fireball simulation with elastic conversion processes described by rate equations. The details of this calculation can be found in Ref. [7]. The $K$ factor for the elastic parton cross sections is kept variable to illuminate different scenarios: $K=0$ (no conversions), $K=1$ (conversions dominated by elastic processes), and $K=4$ (mimicking conversions subject to much stronger coupling than given by elastic scattering). It can be seen from Fig. 1 that conversions into photons are consistent with the observed photon yield. Conversions between quarks and gluons with large $K$ factor are needed to avoid a larger relative suppression of protons.

It was pointed out in Ref. [7] that strangeness at RHIC energies could be the perfect observable to measure conversions of leading jet partons. The bigger picture here is as follows. It is reasonable to believe that strange quarks are chemically equilibrated in the quark gluon plasma at RHIC, with a ratio of strange to light quarks, $w_{\text {med }}=s /(u+d) \approx 0.5$. On the other hand, at RHIC energies, initial jet production at intermediate and large momenta proceeds mostly through Compton scattering of 



Figure 3: Left panel: The azimuthal asymmetry $v_{2}$ for light quarks, strange quarks and gluons without conversions. Right panel: the same with conversions. The $v_{2}$ for light quarks and gluons is not similar, while strange quarks exhibit a suppression.

quarks out of the initial parton distributions, hence suppressing strange quarks in the initial jet sample, $w_{\text {jet }}<0.1$. This large chemical imbalance in the jet sample has an opportunity to equilibrate through their coupling to the quark gluon plasma. Indeed, the ratio of strange to light quarks in the jet sample increases significantly if conversions are allowed. This translates naturally into an enhancement of kaons over pions - or actually into less suppression of kaons vs pions in the nuclear modification factor $R_{A A}$. Fig. 2 shows the change in $R_{A A}$ for neutral kaons expected with conversions.

This mechanism is obscured at LHC energy where the initial jet sample is already close to equilibrium due to the dominance of the $g+g$ fusion channel in initial jet production. It also turns out that heavy quarks can not play the same role as conversion signatures at LHC that strangeness holds at RHIC. The obstacle is that charm quarks will not equilibrate chemically in the quark gluon plasma at LHC. With both low- and high- $p_{T}$ charm quarks produced perturbatively in initial hard scatterings the chemical gradient is not large enough to see a noticable enhancement at high $p_{T}$ [20].

Yet another signature for conversions of high $p_{T}$ particles in the medium should be seen in their azimuthal asymmetry $v_{2}$. It had first been realized for photons [21] that conversions lead to a higher conversion yield on the thicker side of the medium. This is opposite to the suppression driven $v_{2}$ which would lead to less yield on the thicker side of the medium. Hence a conversion driven azimuthal asymmetry should lead to negative $v_{2}$. Of course, all sources of a given particle have to be added up for the total yield, leading to cancellations in $v_{2}$. For direct photons it was forecast that the cancellation is rather complete and that $v_{2}$ should be numerically very small $[21,7,22,23]$. This is in accordance with first measurements of direct photon elliptic flow.

We also find that the conversion mechanism leads to a reversed azimuthal asymmetry for additional strange quarks [24] at high $p_{T}$ at RHIC energies. After adding the effect of energy loss the total $v_{2}$ stays positive but is still significantly suppressed compared to light quarks and gluons. This is shown in Fig. 3. Without conversions, light and strange quarks exhibit the same azimuthal asymmetry, while gluons show larger values due to the larger color factor (left). With conversions 


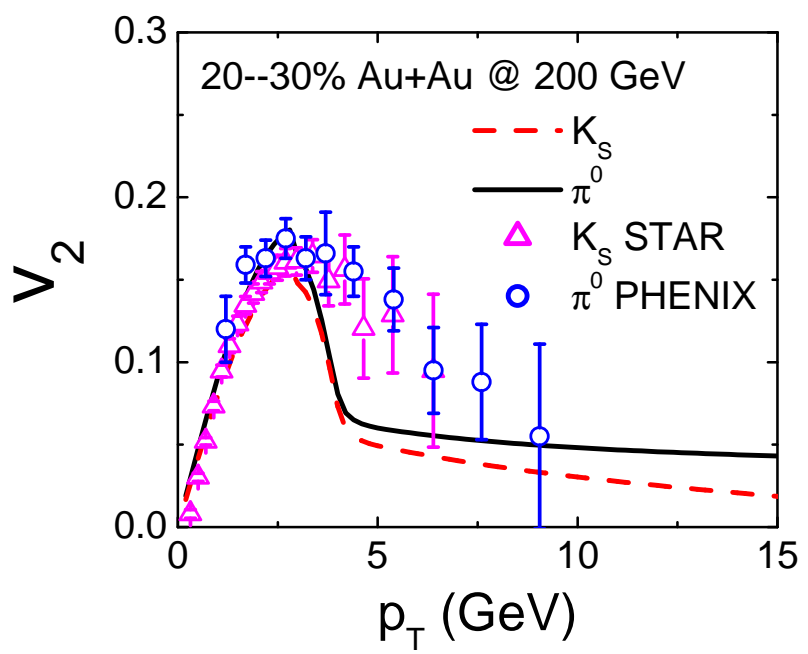

Figure 4: The resulting azimuthal asymmetry $v_{2}$ for kaons which is expected to be suppressed compared to that of pions. Data from $[25,26]$.

enabled, light quarks and gluons exhibit comparable $v_{2}$ while the asymmetry for strange quarks is suppressed (right). Details of the calculation can be found in [24]. Finally, Fig. 4 shows the effects translated into the $v_{2}$ for neutral kaons. It is expected that their $v_{2}$ shows a noticable suppression starting at about 6 to $7 \mathrm{GeV} / c$ compared with pions.

To summarize, we have argued that conversions of leading jet particles is a natural consequence of their interactions with a surrounding medium. In particular, we expect additional photons and strange hadrons at RHIC energies. While the new photon source will increase in relative brightness at LHC we predict the reduced kaon suppression to dissappear at the larger collider energy. $v_{2}$ suppression at large $p_{T}$ for both photons and kaons could provide additional insight. Measuring effects from conversion processes could provide complementary information about the mean free path of fast partons in quark gluon plasma.

RJF would like to thank the organizers of the Workshop High- $p_{T}$ physics at LHC for their kind invitation.

\section{References}

[1] X. N. Wang and M. Gyulassy, Gluon shadowing and jet quenching in $A+A$ collisions at $s^{1 / 2}=200$ GeV, Phys. Rev. Lett. 68, 1480 (1992); M. H. Thoma and M. Gyulassy, Energy loss of high-energy quarks and gluons in the quark - gluon plasma, Nucl. Phys. A 544, 573C (1992).

[2] R. Baier, Y. L. Dokshitzer, A. H. Mueller, S. Peigne and D. Schiff, Radiative energy loss and $p(T)$-broadening of high energy partons in nuclei, Nucl. Phys. B 483, 291 (1997), [arXiv:hep-ph/9608322]; Nucl. Phys. B 484, 265 (1997), [arXiv:hep-ph/9607355].

[3] B. G. Zakharov, Fully quantum treatment of the Landau-Pomeranchuk-Migdal effect in QED and QCD, JETP Lett. 63, 952 (1996), [arXiv:hep-ph/9607440].

[4] U. A. Wiedemann, Jet quenching versus jet enhancement: A quantitative study of the BDMPS-Z gluon radiation spectrum, Nucl. Phys. A 690, 731 (2001), [arXiv:hep-ph/0008241]. 
[5] M. Gyulassy, P. Levai and I. Vitev, Non-Abelian energy loss at finite opacity, Phys. Rev. Lett. 85, 5535 (2000), [arXiv:nucl-th/0005032]; Reaction operator approach to non-Abelian energy loss, Nucl. Phys. B 594, 371 (2001), [arXiv:nucl-th/0006010].

[6] P. Arnold, G. D. Moore and L. G. Yaffe, Photon and gluon emission in relativistic plasmas, JHEP 0206, 030 (2002), [arXiv:hep-ph/0204343]; S. Jeon and G. D. Moore, Energy loss of leading partons in a thermal QCD medium, Phys. Rev. C 71, 034901 (2005), [arXiv:hep-ph/0309332].

[7] W. Liu and R. J. Fries, Probing Nuclear Matter with Jet Conversions, Phys. Rev. C 77, 054902 (2008), [arXiv:0801.0453 [nucl-th]].

[8] R. J. Fries, B. Müller and D. K. Srivastava, High energy photons from passage of jets through quark gluon plasma, Phys. Rev. Lett. 90, 132301 (2003), [arXiv:nucl-th/0208001].

[9] R. J. Fries, B. Müller and D. K. Srivastava, Centrality dependence of direct photons in $A u+A u$ collisions at $s(N N)^{1 / 2}=200 \mathrm{GeV}$, Phys. Rev. C 72, 041902 (2005), [arXiv:nucl-th/0507018].

[10] D. K. Srivastava, C. Gale and R. J. Fries, Large mass dileptons from the passage of jets through quark gluon plasma, Phys. Rev. C 67, 034903 (2003), [arXiv:nucl-th/0209063]; S. Turbide, C. Gale, D. K. Srivastava and R. J. Fries, High momentum dilepton production from jets in a quark gluon plasma, Phys. Rev. C 74, 014903 (2006), [arXiv:hep-ph/0601042].

[11] C. Gale, T. C. Awes, R. J. Fries and D. K. Srivastava, The electromagnetic signature of jets, J. Phys. G 30, S1013 (2004), [arXiv:nucl-th/0403054].

[12] S. Turbide, C. Gale, S. Jeon and G. D. Moore, Energy loss of leading hadrons and direct photon production in evolving quark-gluon plasma, Phys. Rev. C 72, 014906 (2005), [arXiv:hep-ph/0502248].

[13] S. Turbide, C. Gale, E. Frodermann and U. Heinz, Electromagnetic radiation from nuclear collisions at RHIC energies, arXiv:0712.0732 [hep-ph].

[14] W. Liu, C. M. Ko, and B. W. Zhang, Jet conversions in a quark-gluon plasma, Phys. Rev. C 75, 051901 (2007), [arXiv:nucl-th/0607047]; Jet conversions in QGP and suppression of identified hadrons, Int. J. Mod. Phys. E 16, 1930 (2007).

[15] C. M. Ko, W. Liu and B. W. Zhang, Jet flavor conversions in the quark-gluon plasma, Few Body Syst. 41, 63 (2007).

[16] A. Schäfer, X. N. Wang and B. W. Zhang, Multiple Parton Scattering in Nuclei: Quark-quark Scattering, Nucl. Phys. A 793, 128 (2007), [arXiv:0704.0106 [hep-ph]].

[17] S. S. Adler et al. [PHENIX Collaboration], Centrality dependence of direct photon production in $s(N N)^{1 / 2}=200 \mathrm{GeV} A u+$ Au collisions, Phys. Rev. Lett. 94, 232301 (2005), [arXiv:nucl-ex/0503003].

[18] S. Albino, B. A. Kniehl and G. Kramer, Fragmentation functions for light charged hadrons with complete quark flavour separation, Nucl. Phys. B 725, 181 (2005), [arXiv:hep-ph/0502188].

[19] B. I. Abelev et al. [STAR Collaboration], Identified baryon and meson distributions at large transverse momenta from $\mathrm{A} u+$ Au collisions at $s(N N)^{1 / 2}=200 \mathrm{GeV}$, Phys. Rev. Lett. 97, 152301 (2006), [arXiv:nucl-ex/0606003].

[20] W. Liu and R. J. Fries, Heavy Quark Production from Jet Conversions in a Quark-Gluon Plasma, Phys. Rev. C 78, 037902 (2008), [arXiv:0805.1093 [nucl-th]].

[21] S. Turbide, C. Gale and R. J. Fries, Direct photon elliptic flow in high energy nuclear collisions, Phys. Rev. Lett. 96, 032303 (2006), [arXiv:hep-ph/0508201]. 
[22] R. Chatterjee, E. S. Frodermann, U. W. Heinz and D. K. Srivastava, Elliptic flow of thermal photons in relativistic nuclear collisions, Phys. Rev. Lett. 96, 202302 (2006), [arXiv:nucl-th/0511079]; R. Chatterjee, D. K. Srivastava, U. W. Heinz and C. Gale, Elliptic flow of thermal dileptons in relativistic nuclear collisions, Phys. Rev. C 75, 054909 (2007), [arXiv:nucl-th/0702039].

[23] B. Z. Kopeliovich, A. H. Rezaeian and I. Schmidt, Azimuthal Asymmetry of Prompt Photons in Nuclear Collisions, Nucl. Phys. A 807, 61 (2008), [arXiv:0712.2829 [hep-ph]].

[24] W. Liu and R. J. Fries, Elliptic Flow of Rare High-Momentum Probes in Nuclear Collisions, arXiv:0805.3721 [nucl-th].

[25] D. Winter, PHENIX measurement of particle yields at high $p(T)$ with respect to reaction plane in $A u+$ Au collisions at $s^{1 / 2}=200 \mathrm{GeV}$, Nucl. Phys. A 774, 545 (2006), [arXiv:nucl-ex/0511039].

[26] B. I. Abelev et al. (STAR Collaboration), Centrality dependence of charged hadron and strange hadron elliptic flow from $\sqrt{\left(s_{N N}\right)}=200 \mathrm{GeV} A u+A u$ collisions, Phys. Rev. C 77, 054901 (2008), [arXiv:0801.3466 [nucl-ex]]. 